\title{
Impairment of cognitive function by chemotherapy: association with the disruption of phase-locking and synchronization in anterior cingulate cortex
}

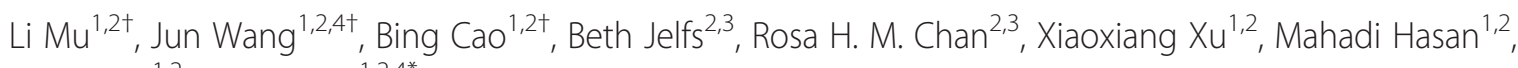
Xu Zhang ${ }^{1,2}$ and Ying Li ${ }^{1,2,4^{*}}$

\begin{abstract}
Background: Patients following prolonged cancer chemotherapy are at high risk of emotional and cognitive deficits. Research indicates that the brain neuronal temporal coding and synaptic long-term potentiation (LTP) are critical in memory and perception. We studied the effects of cisplatin on induction of LTP in the basolateral amygdala (BLA)-anterior cingulate cortex (ACC) pathway, characterized the coordination of spike timing with local theta oscillation, and identified synchrony in the BLA-ACC network integrity.

Results: In the study presented, the impacts of cisplatin on emotional and cognitive functions were investigated by elevated plus-maze test, Morris water maze test, and rat lowa gambling task (RGT). Electrophysiological recordings were conducted to study long-term potentiation. Simultaneous recordings from multi-electrodes were performed to characterize the neural spike firing and ongoing theta oscillation of local field potential (LFP), and to clarify the synchronization of large scale of theta oscillation in the BLA-ACC pathway. Cisplatin-treated rats demonstrated anxiety- like behavior, exhibited impaired spatial reference memory. RGT showed decrease of the percentage of good decision-makers, and increase in the percentage of maladaptive behavior (delay-good decision-makers plus poor decision-makers). Cisplatin suppressed the LTP, and disrupted the phase-locking of ACC single neural firings to the ongoing theta oscillation; further, cisplatin interrupted the synchrony in the BLA-ACC pathway.

Conclusions: We provide the first direct evidence that the cisplatin interrupts theta-frequency phase-locking of ACC neurons. The block of LTP and disruption of synchronized theta oscillations in the BLA-ACC pathway are associated with emotional and cognitive deficits in rats, following cancer chemotherapy.
\end{abstract}

Keywords: Cisplatin, Decision-making, Learning and memory, Synaptic plasticity, Theta oscillation

\section{Introduction}

The 'chemobrain' refers to a range of cognitive deficits caused by prolonged chemotherapy. In clinical studies, depression and cognitive impairment were found in 40 to $70 \%$ of patients following cancer therapy [1]. Cisplatin, a platinum compound, is an anticancer drug

\footnotetext{
*Correspondence: yingli@cityu.edu.hk

${ }^{\dagger}$ Equal contributors

'Department of Biomedical Sciences, City University of Hong Kong, Tat Chee Avenue, Kowloon, Hong Kong

${ }^{2}$ Centre for Biosystems, Neuroscience, and Nanotechnology, City University of Hong Kong, Kowloon, Hong Kong

Full list of author information is available at the end of the article
}

widely used for the chemotherapy of different malignancies. It has been shown in non-human primates that cisplatin can penetrate the Blood Brain Barrier (BBB) and accumulate in the cerebrospinal fluid of the brain [2], affect several neurobiological processes $[3,4]$ including oxidative stress [5]. At the cellular level, chemotherapeutic agents target the central nervous system (CNS) proliferating cells as well as changing fate decisions and cellular functions of neural progenitor cells [3]. Considering that these agents are potent neurotoxins [6], they may contribute to neurobiopathlogical processes, such as apoptosis and cell death correlated with platinum- 
DNA binding $[6,7]$, which are involved in the progress of cognitive deficiency following chemotherapy [8]. At the synaptic level, activity-dependent plasticity in synaptic strength, such as long-term potentiation (LTP), is a key mechanism in shaping cortical circuits $[9,10]$. Therefore, the study of the effect of cisplatin treatment on the modulation of synaptic transmission in animal model may explain its influence on cognitive function.

The anterior cingulated cortex (ACC) is a major cortical area of the limbic loop system, integrating emotion and cognition. The anatomic connections between the ACC and the amygdala have been clearly reported previously $[11,12]$. Indeed, an indirect linkage in the ACCthalamus-amygdala circuitry $[13,14]$ plays an important role in synchronized function between the thalamus and amygdala, and ACC [15]. However, the synaptic metaplasticity in the amygdala-ACC circuitry following prolonged chemotherapy has not been fully explored. Therefore, we sought to characterize if chemotherapy disrupts LTP in the amygdala-ACC network integrity, which is associated with mood disorder and deficits of learning and memory.

Coordinated action-potential timing across populations of neurons is necessary for induction of synaptic plasticity [16]. Recent neurobiological studies of human memory and perception share common observations of theta frequency band oscillations in the brain and coherency of the theta oscillations and action potential activity. Rutishauser et al. have shown that memory formation in humans is predicted by close coordination of spike timing with the local theta oscillation [17]. This suggests that synchronized oscillatory activity promotes the communication between anatomically distant, yet functionally related, structures during learning.

In previous animal research chemotherapy-related mood and cognitive deficits have not been investigated systematically [8]. Our goal in the present study was to go beyond previous animal work, by characterizing the alteration of phase-locking of neural activity to the theta oscillation. And as such allowing identification of the disruption of the amygdala-ACC network synchrony associated with impairment of cognition and behavior after chemotherapy. In order to accomplish this we performed a series of behavioral assessments in rats following chronic administration of cisplatin, in particular the rat Iowa gambling task (RGT) was used to assess decision making functions in rats [18]. To characterize synaptic plasticity in vivo, we recorded evoked local field potentials (LFP) and induced LTP in the basolateral amygdala (BLA)-ACC pathways. Simultaneous recording from an array of microelectrodes allows characterization of the disruption to the phase-locking of single neural spike firing with the ongoing theta oscillation of LFP in a large scale population of neurons in the ACC and identifying the desynchronized theta activity between BLA and ACC following administration of cisplatin.

\section{Results}

\section{Cisplatin suppressed spontaneous exploratory activity}

To examine if cisplatin-treated rats develop anxiety-like behavior we performed open field test (OFT). The OFT is often used to assess exploration in a novel environment and offer a preliminary screen for anxiety-related behavior in rats [19].

Cisplatin-treated rats exhibited a lower total horizontal distance traveled (horizontal activity; $\mathrm{t}=2.54, \mathrm{p}<0.05$ ), a reduced number of rearings (vertical activity; $\mathrm{t}=4.08, \mathrm{p}<$ $0.001)$, a reduced time spent in $(\mathrm{t}=3.01, \mathrm{p}<0.01)$ and number of entries into the center $(t=3.29, p<0.01)$ during the 5-min testing session (Fig. 1a, b, c, d). In contrast to the controls, rats with cisplatin treatment exhibited strongly increased freezing behavior $(t=4.21, \mathrm{p}<0.001$; Fig. 1h).

\section{Anxiety-like behavior}

To confirm the anxiety-like behavior we performed elevated plus-maze (EPM) test. The EPM is a popular behavioral test for anxiety-like behavior and is thought to result from natural aversion of rats to explore elevated and open areas [20].

The EPM tests showed that cisplatin-treated rats exhibited a reduced time in the open arms $(t=3.07, \mathrm{p}<$ 0.01 ; Fig. 1e) and a lower percentage of time spent in the open arms versus closed arms $(t=2.79, \mathrm{p}<0.01$; Fig. 1f) when compared with control rats, suggesting increased anxiety-like behavior induced by chronic cisplatin treatment. As previously observed in the open field test (OFT), rats with cisplatin also exhibited a higher level of freezing behavior during exposure to the elevated plus-maze (EPM) $(\mathrm{t}=2.71, \mathrm{p}<0.05$; Fig. 1g).

\section{Spatial reference recent and remote memory}

To investigate the learning acquisition processes and memory ability we conducted Morris water maze task [21]. The test used was described by Kesner [22] as a test of prefrontal cortex (PFC) function. Its goal is to assess the ability of rats to learn the position of the hidden platform and to keep this information online during four consecutive trials.

Swimming speed did not change in cisplatin-treated rats during probe training (acquisition trials) in Morris water maze (MWM). Escape latencies were used to indicate the ability to learn the location of a submerged platform using visual cues. There was a significant effect on escape latencies for groups $(F(1,31)=8.03$ and $F$ $(1,24)=8.87$; both $\mathrm{p}<0.01$ for recent and remote memory), for trials $(F(3,31)=35.35$ and $F(3,24)=41.59$; both $\mathrm{p}<0.001)$ for recent and remote memory), and for their interaction $(F(3,93)=3.80$ and $F(3,72)=2.85$; both $\mathrm{p}<0.05$ for recent and remote memory). These results 


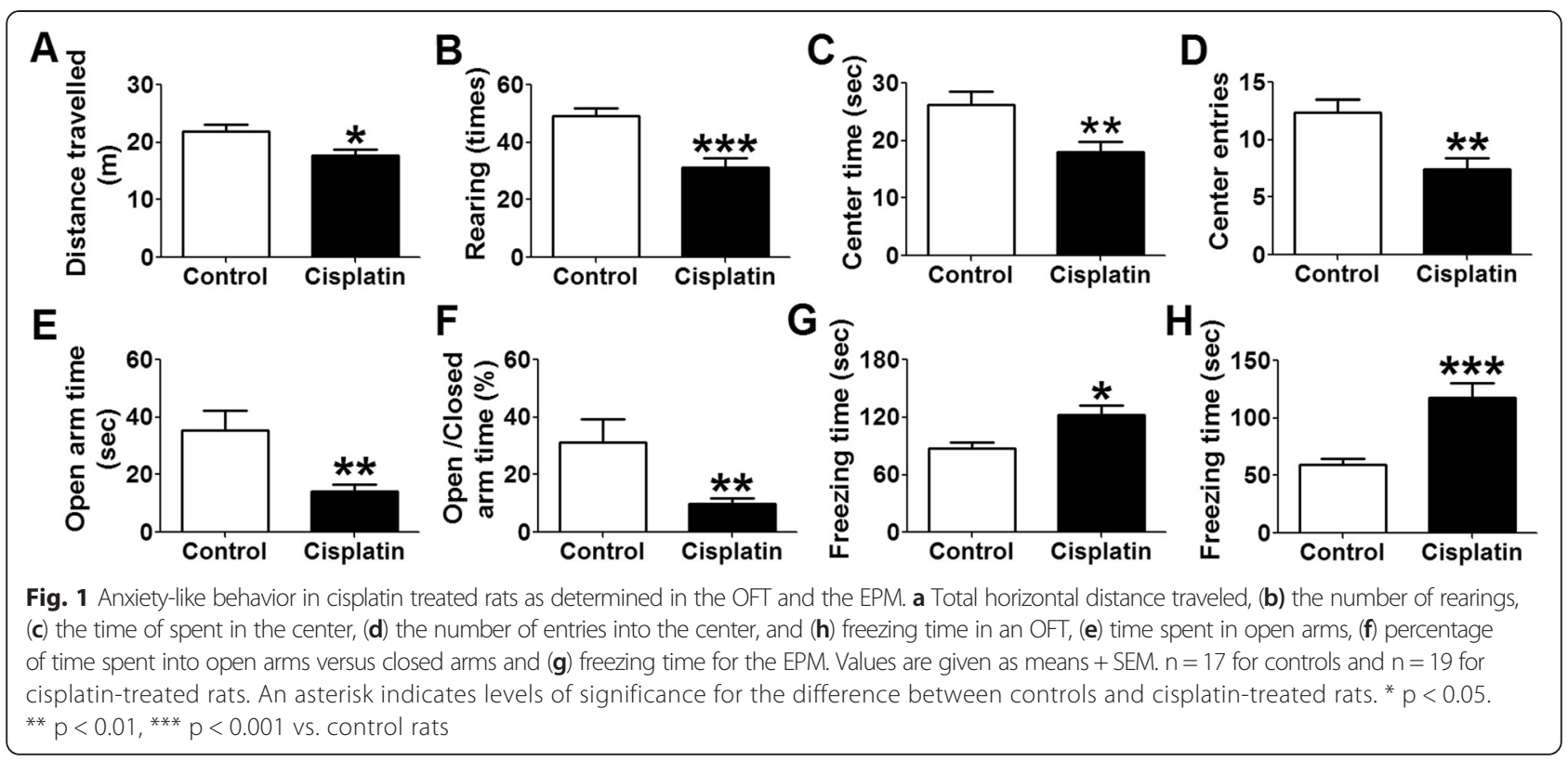

suggest that cisplatin-treated rats spent more time trying to find the platform during 4-day acquisition over the course of several trials (Fig. 2a and b). Spatial memory was assessed in a single $60 \mathrm{~s}$ probe test in which rats searched the target quadrant that previously contained the escape platform. Control animals showed a significantly higher percentage of time spent in the target quadrant compared with cisplatin rats when tested in a probe trial 24-h after training $(t=3.33, \mathrm{p}<0.01$; Fig. $2 \mathrm{c})$ and in 30 day after training $(t=3.61, p<0.01$; Fig. 2d), suggesting
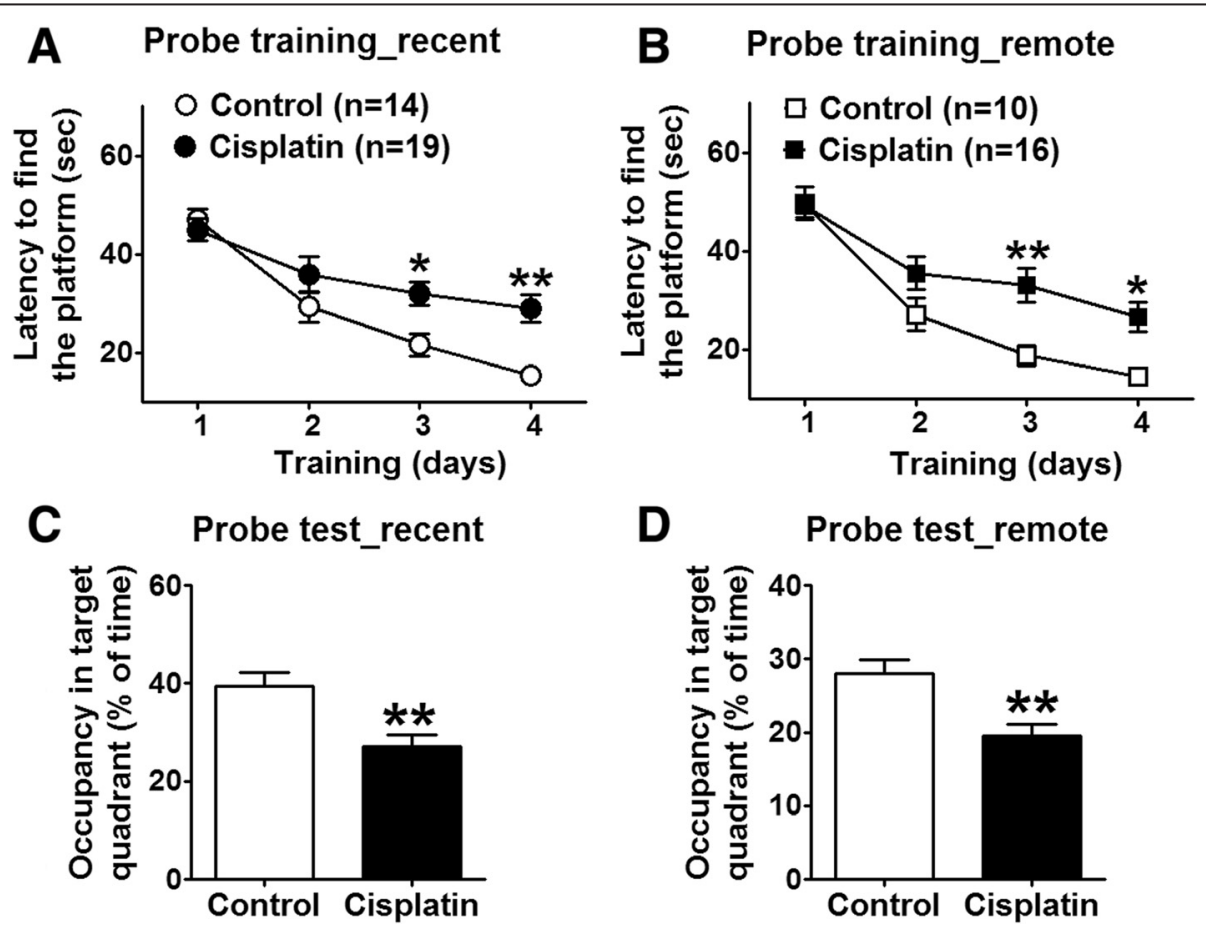

D

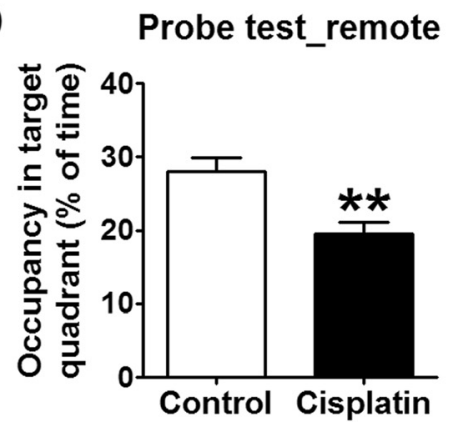

Fig. 2 Spatial reference learning and memory impairment observed in cisplatin-treated rats exposed to the water maze test. $\mathbf{a}$ - $\mathbf{b}$ In the probe training sessions, the acquisition curve of cisplatin rats was impaired compared with control rats. $\mathbf{c}-\mathbf{d}$ In 60 s probe tests, cisplatin-treated rats spent significantly lower percentage of time in the quadrant previously containing the platform than control rats, suggesting impaired memory. An asterisk indicates levels of significance for the difference between controls and cisplatin-treated rats.* $p<0.05,{ }^{* *} p<0.01$ 
cisplatin-disrupted memory retrieval in both the recent and remote memory retention.

\section{Cisplatin-induced behavioral changes in decision-making during the rat lowa gambling task}

The rat gambling task (RGT) has been developed to test the decision-making capacities in rats via a conflict between immediate and long-term gratification (food reward) [23].

During the RGT different subgroups of decision-making performers were identified in both the control and cisplatin-treated rats, based on the percentage of advantageous choices (choice $\mathrm{C}$ and $\mathrm{D}$ ) made during the testing session (Fig. 3a and b). During the first $10 \mathrm{~min}$ of the 60 min testing session, no obvious choice preference was observed in any subgroup in either the control or cisplatin group (Fig. 3a and b). Over time, some rats gradually preferred advantageous choices and finally developed good decision-making behavior (defined by advantageous choices $>70 \%$ in the last $20 \mathrm{~min}$ ). Delayed good decision making behavior was identified when the percentage of advantageous choices in good decision-makers was below $70 \%$ at $30 \mathrm{~min}$. Poor decision-making corresponded to those with less than $30 \%$ advantageous choices in the last $20 \mathrm{~min}$. The remaining rats showed no obvious preference for either advantageous or disadvantageous choices throughout the test (between $30 \%$ and $70 \%$ preference for advantageous choices in the last $20 \mathrm{~min}$ ), and were classified as undecided. The proportion of delayed-good decision-makers increased after repeated cisplatin treatments ( 8 out of 35 cisplatin rats, $23 \%$ ) compared to the control group (2 out of 24 control rats, $8 \%$ ), and the proportion of normal good decision makers was reduced, to $20 \%$ in cisplatin rats compared to $55 \%$ in controls (Fig. $3 \mathrm{c}$ and Table 1). The proportion of undecided performers in the cisplatin group was similar to that observed in controls ( $23 \%$ vs. $20 \%$, cisplatin vs. control), with $34 \%$ and $17 \%$ poor decision-makers observed in the cisplatin and control groups respectively (Fig. 3c and Table 1). The difference in the proportions of the four subgroups between the control and cisplatin groups was significant (non-parametric Mann-Whitney test, $\mathrm{z}=-2.27, \mathrm{p}<0.05$, Fig. 3c), suggesting that cisplatin treatment affects decisionmaking ability in rats

\section{Impaired LTP-like synaptic plasticity}

In all rats, a range of stimuli (50 to $1000 \mu \mathrm{A})$ to the BLA elicited gradually increased ACC evoked LFP (Fig. 4a). No significant difference was observed between control and cisplatin rats $\mathrm{I} / \mathrm{O}$ curves $(\mathrm{F}=0.33, \mathrm{p}>0.05$, Fig. $4 \mathrm{~b})$. In control rats $(\mathrm{n}=5)$, after three trains of TBS delivered to the BLA, a $158.4 \pm 9.6 \%$ enhancement in LFP amplitude in response to BLA stimuli $(500 \mu \mathrm{A})$ was observed compared to the baseline level (pre-TBS). In contrast, TBS failed to induce LTP in cisplatin-treated rats $(n=6)$. Representative evoked LFP are shown in Fig. 4c; there was no obvious alteration of LFP tested in pre-TBS and post-TBS condition at any time points in cisplatin rats $(103.7 \pm 6.4 \%$, Fig. $4 d)$. These results indicate that TBSinduced LTP in the BLA-ACC synapses was impaired after cisplatin administration. We also detected impairment of LTP induction in large-scale ACC in the multiple-channel electrode study (data not shown).
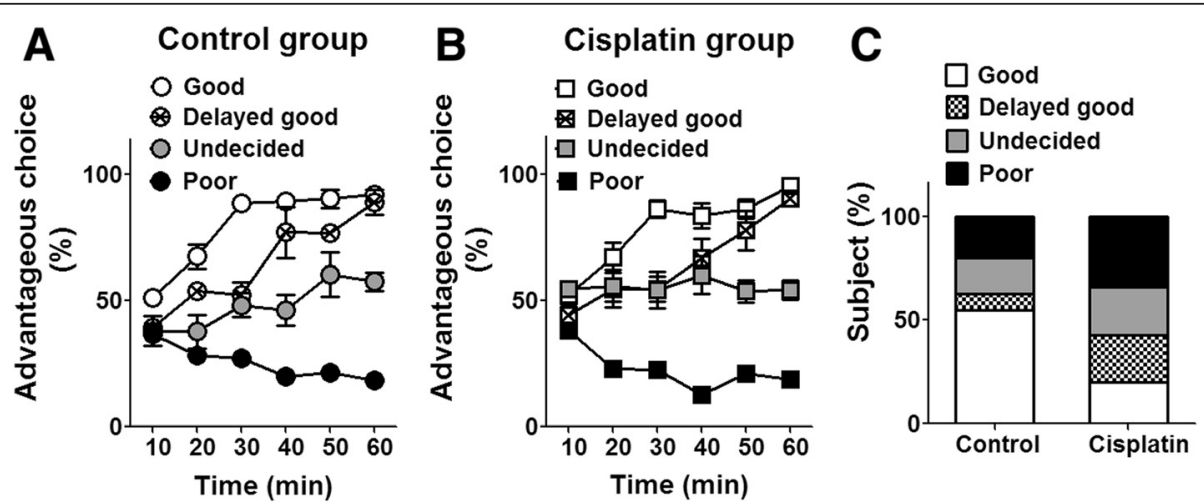

Fig. 3 Changes in decision-making behavior induced by cisplatin using a rat lowa gambling task. a - b Time course of percentage of advantageous choices for good (white), delayed good (grid), undecided (30\% gray) and poor (black) decision-makers during a 60 min RGT testing in control (A circles) and cisplatin (B squares) rats. Within the first $10 \mathrm{~min}$, rats chose equally between advantageous and disadvantageous choices. They then quickly developed four distinct subgroups based on the percentage of advantageous choices during the 60 min test ( $>70 \%$ preference of advantageous choices at 30 min and in the last $20 \mathrm{~min}$ for good decision-maker, $<70 \%$ preference of advantageous choices at $30 \mathrm{~min}$ and $>70 \%$ preference in the last 20 min for delayed good decision-maker, $<30 \%$ in the last 20 min for poor decision-maker, and $30-70 \%$ in the last 20 min for undecided maker). c Proportions of good performers (white bar), delayed good performers (grid bar), undecided behavior (30\% gray bar) and poor decision-making (black bar) for control group and cisplatin group. Advantageous choices $(\%)=$ numbers of nose-poke for choices $(C+D) /$ numbers of nose-poke for choices $(A+B+C+D) * 100 \%$. n = 24 for control group, $\mathrm{n}=35$ for cisplatin group 
Table 1 Number and percentage of individuals exhibiting the behavior observed during the RGT for both the control and cisplatin groups

\begin{tabular}{lll}
\hline Number of individuals & Control group $(n=24)$ & Cisplatin group $(n=35)$ \\
& $n(\%)$ & $n(\%)$ \\
\hline Good decision makers & $13(55 \%)$ & $7(20 \%)$ \\
Delayed-good decision-makers & $2(8 \%)$ & $8(23 \%)$ \\
Poor decision-makers & $4(17 \%)$ & $12(34 \%)$ \\
Undecided decision-makers & $5(20 \%)$ & $8(23 \%)$ \\
\hline
\end{tabular}

\section{Enhanced ACC theta activities}

To characterize the theta rhythm activity of the ACC, power spectral analysis was performed during $30 \mathrm{~s}$ spontaneous and $30 \mathrm{~s}$ CRD stimulation in two groups of rats. The AUC of the theta band power are shown in Fig. 5b ( $\mathrm{n}=7$ for control and 7 for cisplatin group), a significant alteration between the groups was detected ( $\mathrm{F}=7.71$; $\mathrm{p}$ $<0.001)$. Post hoc analysis revealed that the ACC theta power during both spontaneous activity $(\mathrm{t}=3.32$; $\mathrm{p}<$ 0.05 ) and visceral pain condition $(60 \mathrm{mmHg} C R D ; t=$ 3.28; $\mathrm{p}<0.05)$ were enhanced in cisplatin rats compared with control. In control rats the ACC theta power was $3.58 \pm 0.64$ and $4.17 \pm 0.55 \times 10^{-4} \mathrm{mV}^{2}$, while in cisplatin rats the ACC theta power were $6.10 \pm 0.37$ and $6.84 \pm$ $0.60 \times 10^{-4} \mathrm{mV}^{2}$ in spontaneous conditions and during CRD stimulation respectively. Theta/delta ratios were also evaluated (Fig. 5d); post hoc analysis revealed that in cisplatin-treated rats under spontaneous conditions, ACC theta/delta ratio was enhanced from $8.50 \pm 0.80$ to 13.77 $\pm 1.08 \%(\mathrm{t}=2.91 ; \mathrm{p}<0.05)$. And during CRD stimulation, the theta/delta ratio was increased from $12.85 \pm 1.44$ to $19.94 \pm 1.60 \%(t=4.08 ; \mathrm{p}<0.05)$. These data indicate that cisplatin enhances ACC theta activities.

\section{Disrupted spikes phase-locking to theta oscillation in the ACC in cisplatin-treated rats}

To further investigate the strength of coupling between spike timing and LFP at any given frequency, we next asked whether spike phase distributions in the local theta oscillation of local field potential were changed in rats after cisplatin treatment. As expected, we found that in control rats $44.2 \%$ of neurons (53 of 120 neurons) fired spikes that were phase-locked to the LFP oscillations in theta range (Fig. 6a, p $<0.0023,0.05 / 22$, Rayleigh's test). The preferred

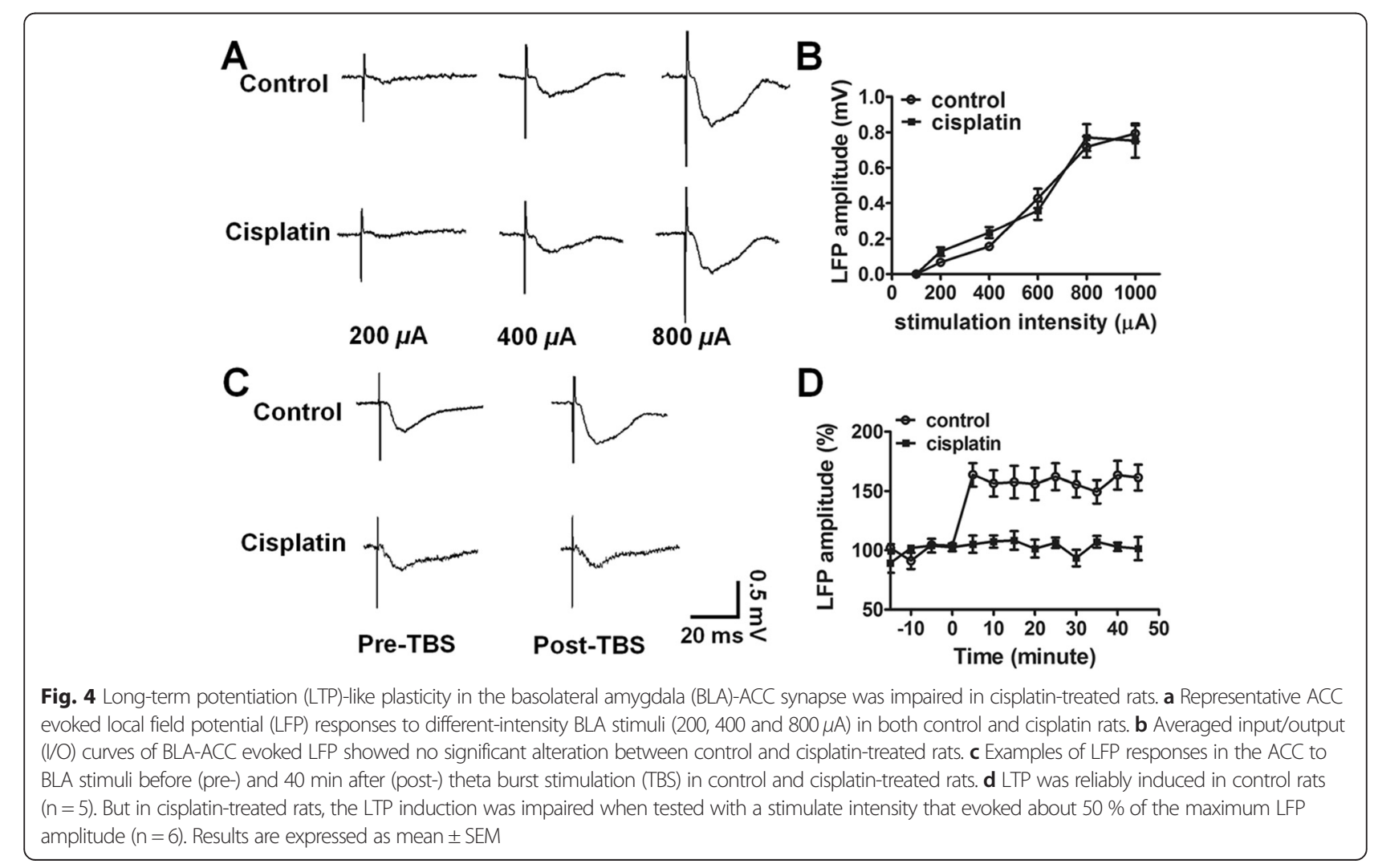




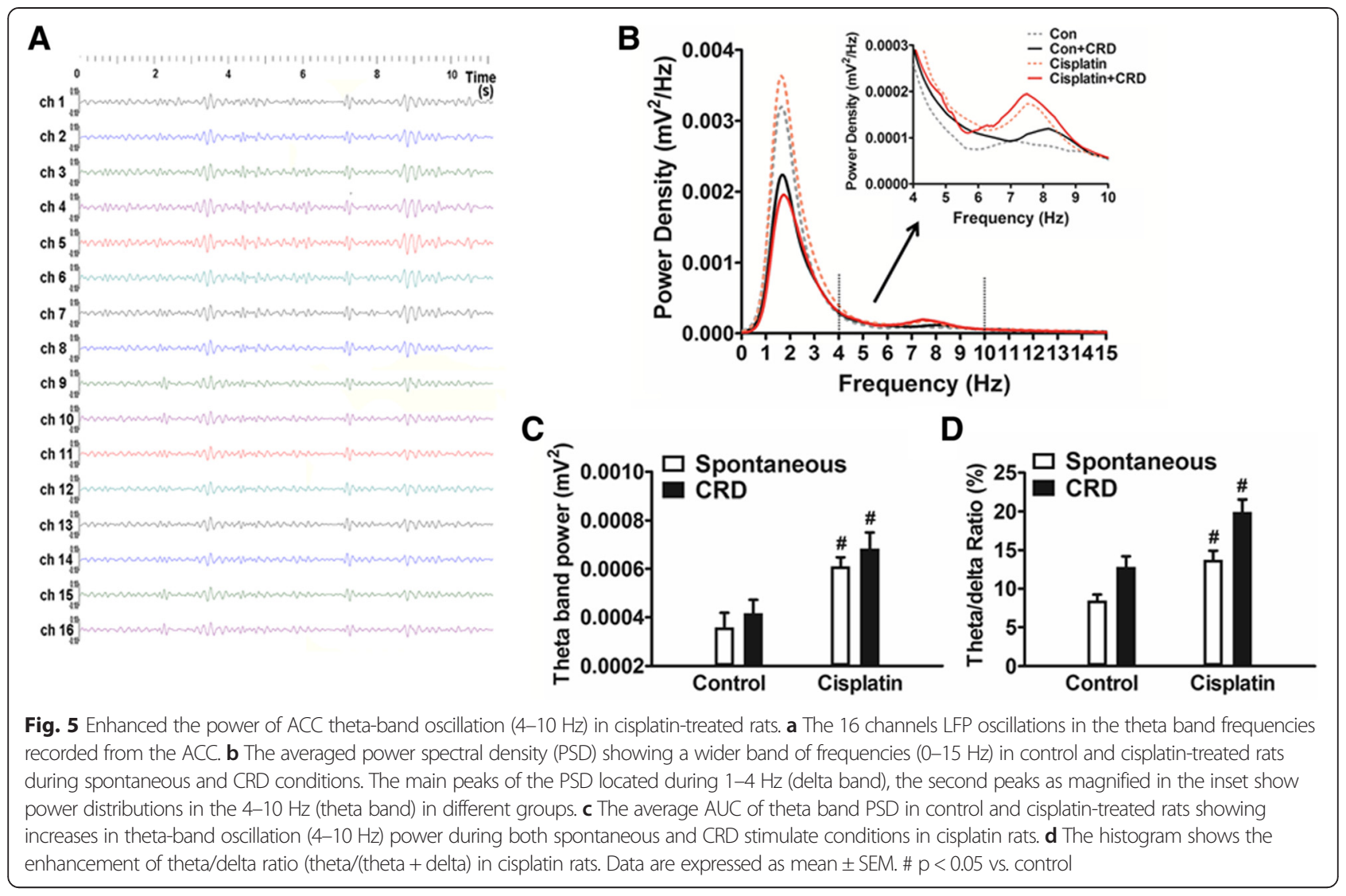

frequency of phase-locked neurons was $8.03 \pm 0.98 \mathrm{~Hz}$. In contrast, in cisplatin group, only $12.8 \%$ (19 of 149 neurons) showed phase-locking at theta range. In control rats the neurons had a range of phase preferences, with the majority (27 of 53 neurons, $51 \%$ ) firing close to the trough of the theta band oscillation $\left( \pm 45^{\circ}\right.$ around trough; Fig. 6b). Examples of phase distributions of a phase-locked neuron in a control rat and an un-phaselocked neuron in a cisplatin rat are presented as polarhistograms (Fig. 6c and d). The phase-locked neuron in the control rat showed most spikes firing at between $120^{\circ}$ and $240^{\circ}$ of the theta cycle with a mean-phase of $184^{\circ}$. However, in cisplatin-treated rat the un-phaselocked neuron displayed random firing. Together, the results indicate the disruption of the ACC phase distribution of single neuron spikes in relation to ongoing local field potential in cisplatin rats.

\section{Desynchronized theta activities in BLA and ACC}

To examine the synchronous activities between BLA and ACC we compared theta oscillations of the local field potential at rest and during noxious stimulation in both the control and cisplatin-treated rats. Here, the CRD-induced responses provide an index of how the system responds to activation. The original traces of spontaneous theta activity are shown in Fig. 7a. Time- varying power spectral analysis of $120 \mathrm{~s}$ recordings revealed that the concentrated neuronal activities in the ACC and BLA of control rats, both at rest and during visceral pain stimulation (CRD $30 \mathrm{~s}$ ), became dispersed at theta frequencies in cisplatin rats (Fig. 7b). Crosscorrelation analysis allows quantitative evaluation of the synchronized activity in the LFP. By averaging the cross-correlograms and taking the second positive peak as a quantitative measure (Fig. 7c), a significant difference was detected $(\mathrm{F}=21.35, \mathrm{p}<0.001$; Fig. $7 \mathrm{~d})$. Post hoc analysis revealed that in spontaneous conditions (basal) the correlation values were decreased in cisplatin rats, from $0.147 \pm 0.013$ in control rats $(n=6)$ to 0.090 \pm 0.005 in cisplatin rats $(n=6 ; t=3.93, p<0.05)$. During visceral pain stimulation the cross-correlation values of control rats were increased from $0.147 \pm 0.013$ at basal values to $0.174 \pm 0.010$ during $\operatorname{CRD}(\mathrm{t}=3.42, \mathrm{p}<0.05)$. In contrast, in cisplatin rats visceral pain stimulation failed to alter the cross-correlation value between BLA and ACC $(0.090 \pm 0.010$ of basal vs. $0.085 \pm 0.005$ during $\mathrm{CRD}, \mathrm{t}=2.77, \mathrm{p}>0.05)$. The result suggests desynchronized activity between BLA and ACC in both resting and visceral pain stimulation in cisplatin-treated rats. This indicates that the BLA and ACC may only loosely interact for dynamic information transfer after cisplatin treatment. 

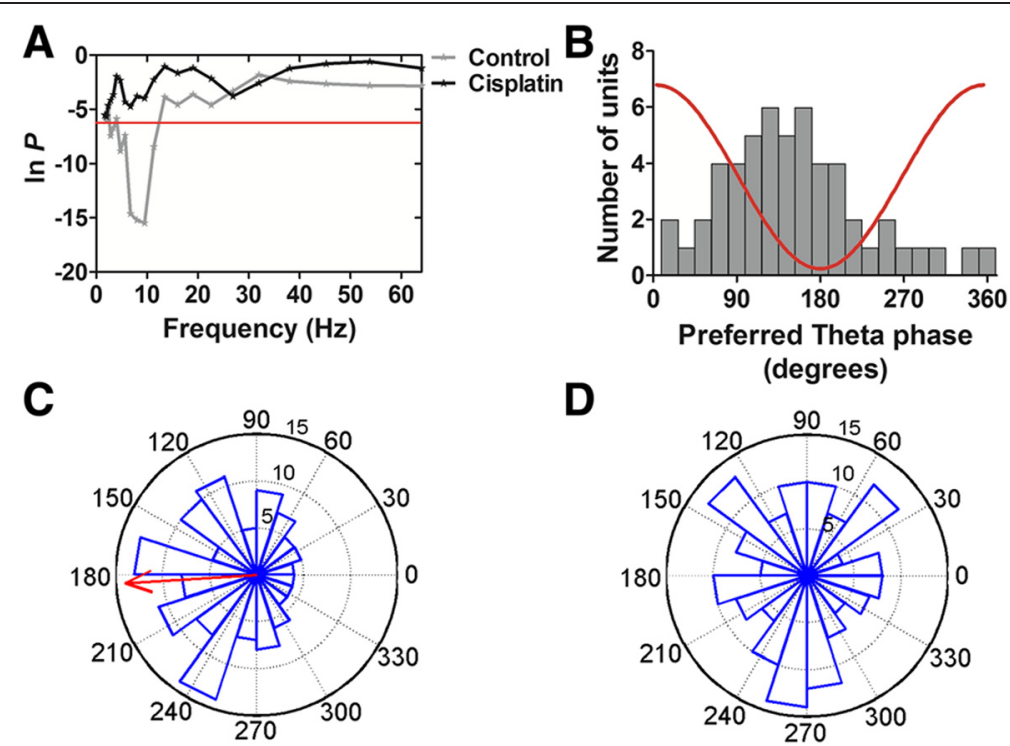

Fig. 6 Cisplatin disrupted the spikes phase-locking in the ACC. a Test of significance of phase-locking as a function of frequency ( 1 - $64 \mathrm{~Hz})$. The threshold (red line) for significant phase-locking was set to $p=0.0023$ (0.05/22, Bonferroni corrected). The shown phase-locked neuron in the control rat (gray line) exhibited maximal phase-locking at $9.5 \mathrm{~Hz}$ while the un-phase-locked neuron in the cisplatin-treated rat (black line) showed no significant phase-locking in theta range. $\mathbf{b}$ Histogram of the preferred phase of all phase-locked neurons in the control rats ( $n=53$ of 120). The figure shows most phase-locked neurons preferred to fire during the descending phase or at the trough of the oscillation. The red line is a schematic of the theta cycle. c The polarhistogram of the spike-field phase distribution of the phase-locked neuron from the control rat shown in (A). The figure shows the majority of spikes of this neuron fired close to $180^{\circ}$. The mean phase shown by the red arrows indicates this neuron preferred firing at $184^{\circ}$ of the theta oscillation. The vector length $R=0.23$. $\mathbf{d}$ Polar-histogram of the spike-field phase distribution of the un-locked neuron from the cisplatin-treated rat shown in (A). The figure shows this neurons action potential firing at random angles of the theta cycle oscillations suggesting disrupted phase-locking in rats following cisplatin treatment. The vector length $R=0.03$

\section{Discussion}

Using a model of chemotherapy associated with emotional and cognitive deficits, this is the first study to investigate the synaptic plasticity characterized by the phase-locking of the neural spike firing with the ongoing theta oscillation of the LFP. We also clarify the synchronization of large scale theta oscillation in the BLA-ACC pathway. Single cytostatic agent cisplatin was used instead of a chemotherapeutic cocktail in a clinical setting. Cognitive functions were examined up to two months post treatment and the animals were free of cancer and other diseases.

In the EPM [20], we observed that cisplatin-treated rats decreased the amount of time they spent in the open arms, and significantly increased their freezing time suggesting the development of anxiety-like behavior. Anxiety is an instinct for promoting adaptive survival by elusion of unnecessary danger. However, excessive anxiety is unfavorable reducing even the behavioral activity that is necessary for adaptation. The amygdala plays a key role in the expression of anxiety or fear, and the medial prefrontal cortex is important for regulating the amygdala-mediated expression of fear [24].

In the spatial reference memory task, the cisplatintreated rats showed impaired learning acquisition processes. Furthermore, these rats spent less time in the platform quadrant compared to control rats in both recent and remote probe test suggesting impaired recent and remote memory.

The hippocampus plays a critical role in spatial memory formation $[8,25]$, however, as these memories mature, they may become more (or even exclusively) dependent on extra hippocampus structures. Previous studies demonstrated that the medial prefrontal cortex (mPFC) specifically the ACC play a key role in the expression of remote spatial memories [26, 27]. Employing a conditional place avoidance test we recently showed, that ACC neuronal activities play a key role in visceral pain aversion memory processing in awake rats [28]. Using a visceral hypersensitive rat model we have demonstrated that ACC hypersensitivity can be observed up to 7 weeks after the initiation of colonic anaphylaxis and is independent of mucosal inflammation. The prolonged ACC sensitization enhanced visceral pain responses by descending facilitate system [29], this suggests triggering of pain memory in the ACC neuronal circuitry [30-32]. More recently, electrophysiological recording showed LTP of local field potential can be reliably induced in the medial thalamus-ACC synapses by TBS condition stimulation in normal rats [33]; however, this canonical LTP induction was occluded in chronic pain state [33]. Interestingly, similar results were observed in the present studies, the data demonstrate for the first time that cisplatin effectively blocks the expression of LTP at amygdala-ACC 


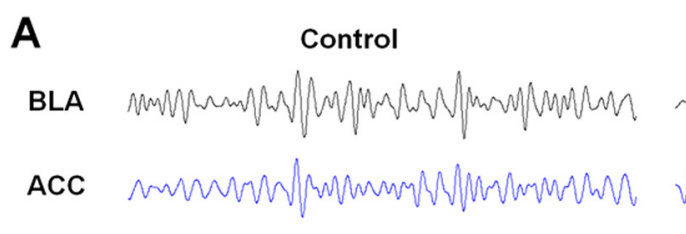

B
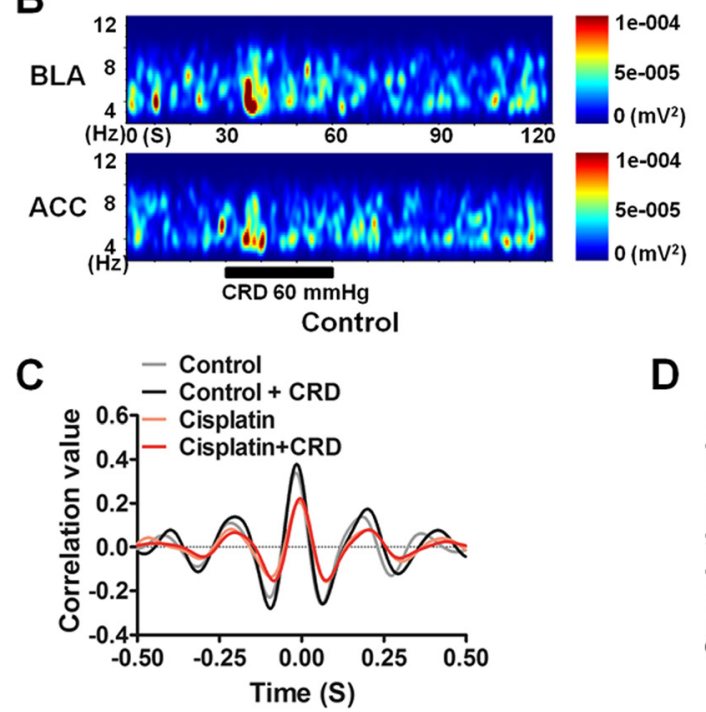

\section{Cisplatin}
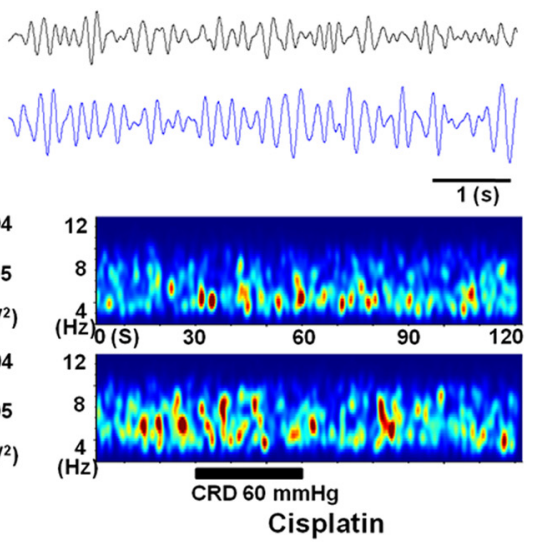

D

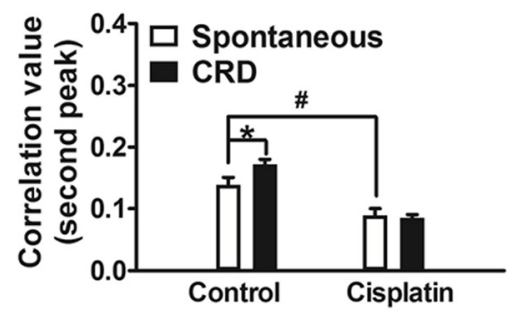

Fig. 7 Desynchronized theta activities between the BLA and ACC in cisplatin rats. a Representative traces of spontaneous theta band field potential in the BLA (black line) and ACC (blue line) in control (left) and cisplatin (right) rats. b Time-varying power spectra of BLA (upper) and ACC (bottom) LFP ${ }_{\theta}$ in control and cisplatin rats. $\mathbf{c}$ The averaged cross-correlograms of control and cisplatin rats for both spontaneous activity and during visceral pain stimulation (60 mmHg CRD). $\mathbf{d}$ Statistical analysis revealed that the cross-correlation value (the second positive peak) was decreased in spontaneous theta activity in cisplatin-treated rats compared with control group. Meanwhile, in control rats, CRD significantly enhanced the correlation value when compared with spontaneous activity. In cisplatin rats there was no changing during CRD stimuli compared with resting state. Data are expressed as mean \pm SEM. ${ }^{*}, \# p<0.05$

synapses in vivo. This occlusion approach has been commonly used to investigate whether the plasticity obtained in vitro by electrical stimulation is a reliable model for studying the mechanisms of learning and memory [34]. These results suggest that chemotherapy agents could causally interrupt learning and memory [8]. In line with the present results, a similar LTP occlusion has been found after a fear paradigm in amygdala [35] and hippocampus [36].

In the brain, there are both small- and large-scale levels of organization [37]. Neural oscillations play a basic role in coordinated activity during normal functional brain processes $[38,39]$. Several lines of evidence suggest that the theta band $(4-10 \mathrm{~Hz})$ in the neural network, which temporally links single neurons into assemblies, cooperatively facilitates synaptic plasticity [40]. Our spectral analysis of ACC local field activities revealed that on average, the cisplatintreated rats exhibited higher spectral power over the frequency range in theta band $(4-10 \mathrm{~Hz})$. However, the frequency at which theta power reached maximum was not significantly shifted. It appears that cisplatin affects the physiological expression of theta oscillation activity, but does not alter the precise frequency of this oscillation. This abnormal theta oscillation power is consistent with previous observations that neurotoxicity of Ecstasy causes an increase in theta power in EEG which is correlated with a decline in attention and memory [41]. An animal study also showed spatial memory deficit accompanied with hippocampal theta power enhancement caused by neurotoxins [42].

A growing body of research evidence suggests that neurons transmit information not only in terms of their firing rates but also by varying the timing of the spikes corresponding to neuronal oscillations [37]. Induction of LTP is favorable when higher frequency electrical stimulations were arrived on the positive phase of theta oscillations [43] Furthermore, behavioral studies in humans and animals have consistently shown that [9] brain oscillations coordinate with timing of single-neuron activity [17], synchronously discharging cells are more effective at driving neurons at subsequent processing stages $[17,44,45]$ than uncoordinatedly responding cells. In addition, to advance the understanding of the timing relationship between spikes and ongoing theta oscillation, it is critical to investigate the angular distributions of spikes with the theta oscillation, and 
clarify the significance of phase-locking of spikes in theta oscillation [17]. We found that in control rats $44.2 \%$ of ACC neurons fired spikes that were phase-locked to the LFP oscillations in the theta range. The preferred frequency of phase-locked neurons was $8.03 \mathrm{~Hz}$, with maximal activity during the descending phase and at the trough of the oscillations. In contrast, only $12.8 \%$ ACC neurons showed phaselocking at theta range in the cisplatin group. Together, these results suggest that chemotherapy disrupts phase distribution of ACC neuron spikes in theta oscillation of LFP.

Decision-making is the result of the integration of several executive brain areas and has emerged as a crucial theme in neurophysiological studies of cognition. Animals are individuals that can exhibit human-like cognitive characteristics, such as the ability to acquire and reason with causal knowledge [46, 47]. Decision making is complex ; it can be impaired in psychiatric disorders, such as attention-deficit/ hyperactivity disorder. The Iowa gambling task (IGT) is frequently used to assess decision-making performance in a clinical setting [48]. Our RGT results show that good, delayed-good, undecided, and poor decision-makers could be identified in the RGT [49]. In the cisplatin-treated group we observed decreases in the percentage of good decisionmakers, and increases in the percentage of maladaptive behavior (delayed-good decision-makers plus poor decisionmakers). Investigators have proved that the ACC plays a crucial role in selecting proper actions when faced with different benefits in an unsure environment [50] through signaling error-likelihood [51]. The ACC also plays a key role in choosing appropriate actions when the environment is dynamic $[52,53]$ related to the information about the costs and benefits [54]. It appears that the dysfunction of the phase-locking of the neural firing with the theta oscillation may contribute to mediate the decision-making deficits following cancer chemotherapy [49]. In addition, and also in agreement with other reports [55], our results revealed that high levels of anxiety were associated with poor decisionmaking using the RGT reward model. This is also in line with human data that highly anxious subjects performed worse on the IGT [56].

Advantageous decision-making in the gambling task also requires an intact amygdala [57], which is a key player in emotions and affective disorders. Accumulating evidence implicates phase synchronization and large-scale integration of neuronal activities in different regions as a mechanism linking functionally related regions of the brain [37, 58]. The direct and indirect connections between ACC and amygdala have been well determined anatomically [11, 13]. Electrical stimulation of the BLA has been shown to change neuronal firing in the $\mathrm{MPFC}$, with latencies consistent with monosynaptic and polysynaptic pathways [59]. Behavioral studies have also shown that the BLA-ACC synaptic pathway participated in various cognitive functions. For example, in chronic pain state, the cognitive impairment was caused by amygdala-driven prefrontal cortical deactivation [60]. Synchronous oscillatory activity in the theta band has been suggested to mediate information flow between functionally related brain regions during learning and memory retrieval [61]. Previous studies have shown that the synchronized activity between frontal and hippocampal regions was modulated by anxiety [62], and enhanced during the execution of working memory tasks using human EEG recording [63]. In the current study, we tested our hypotheses that cisplatin leads to desynchronization in the amygdala and ACC correlated with deficits in learning and memory. By simultaneously recording local field potentials in the ACC and the BLA, cross-correlation and time-varying power spectral analysis of the theta oscillations at rest revealed a pattern of dispersion of theta band activity and reduction in correlation values of cisplatin rats when compared to the control rats. Furthermore, noxious stimulation (CRD) enhanced the synchronized theta activities between BLA and ACC in normal rats but in contrast, non-synchronized theta activities were observed during CRD after cisplatin administration. These data might be particularly relevant to the failure of introduction of LTP in the BLA-ACC pathway after administration of cisplatin. It has been well demonstrated that LTP is induced preferentially on the positive phase of theta oscillations. In cellular studies it was clearer that whether a given stimulus triggers synaptic plasticity can depend on when the stimulus arrives relative to the phase of ongoing theta oscillation [43]. It appears that higher frequency of theta burst stimulation (TBS)-induced LTP shared similar modality with the synchrony of theta oscillation of LFP in the BLA-ACC pathway. We speculate that the theta synchronized activity between circuits might promote the induction of LTP by electrical higher frequency TBS in these circuits. Together these results provide the first clear indication that chemotherapy disrupts the phase-locking of single neural firings with respect to the ongoing theta oscillation, and further interrupts the synchrony in the BLA-ACC pathway, which correlates with blocking LTP induction in this pathway. These observations directly complement recent seminal findings showing that in human studies [17], the interruption of neuronal synchrony appears to impact cognition and behavior.

A limitation of the present study is that electrophysiological studies were performed under anesthesia. We observed an increase in theta/delta ratios after cisplatin treatment. However, another report showed that temozolomide, another drug for chemotherapy, reduced theta/delta ratio in hippocampus of conscious rats after 4 weeks of treatment associated with the disruption of learning [61]. A series of experiments have shown that theta oscillations are quite different among anesthetized and different sleep-wake states in awake rats. Kramis et al. reported two types of theta rhythm in hippocampus, one is sensitive to astropine 
and the other is abolished selectively during ether or urethane induced anesthesia [64]. More recently, other researchers indicated that under anesthesia, hippocampal activity switched from large-amplitude, irregular activity to a theta state, and the theta/slow oscillation ratio increased when transiting anesthetized to the waking, which was mediated by cholinergic [65] and serotonergic neurons [66]. How the neural network between the ACC and amygdala is coordinately activated in the spatial memory and RGT reward models in awaken state remains unexplored. Future studies are clearly needed, using chronic implants of arrays of multi-electrodes and recording from neuronal ensembles during animal behavior. This will enable exploration of the neural mechanisms underlying the functional connectivity between the ACC and amygdala during events involving learning, memory retrieval, and decision-making.

In conclusion these results provide the first direct evidence that cancer chemotherapy can reduce the coherence and interrupt the phase-locking of ACC neuronal spikes with the ongoing theta band of the LFP. More importantly, the disruption of the synchronized theta oscillations and impairment of induction of long-term potentiation of the BLA-ACC pathway are associated with emotional and cognitive deficits in rats following cancer chemotherapy.

\section{Materials and methods}

\section{Animals}

A total of 59 male Sprague-Dawley rats were used in these experiments; they were housed in standard laboratory conditions (temperature at $23 \pm 1{ }^{\circ} \mathrm{C}$ and $12 / 12 \mathrm{~h}$ light-dark cycles with lights on at 7:30 a.m.) The rats were allowed to adapt to their environments for at least 5-7 days before the first injection of cisplatin and were handled for 3-5 min daily for 2-3 days before beginning the behavioral experiments. Rats had free access to water, but had moderate food deprivation during the decision-making testing. All surgical and experimental procedures were conducted according to the guidelines laid down by the NIH in the US regarding the care and use of animals for experimental procedures and were approved by the Committee on Use and Care of Animals at City University of Hong Kong and the licensing authority to conduct experiment from Department of Health of Hong Kong (No. 13-89 in DH/HA\&P/ 8/2/5 Pt.2).

\section{Drug treatments}

The cisplatin dosage and dosing schedule used in the present study were based on the clinical usage of cisplatin treatment into the abdominal cavity in cancer patients, slightly modified according to previous description in rodents [4]. Cisplatin (International Laboratory, USA) was dissolved in $0.9 \% \mathrm{NaCl}(0.4 \mathrm{mg} / \mathrm{ml})$, warmed to $45^{\circ} \mathrm{C}$ and injected with $1.0 \mathrm{ml} / 100 \mathrm{~g}$ rat. The rats weighing 250 $300 \mathrm{~g}$ at the beginning of the experiment received intraperitoneal injections of cisplatin or $0.9 \% \mathrm{NaCl}$ once every 7-8 days for a total of 6 times in recent memory testing group and 8 time for remote memory group (Table 2).

\section{Behavioral assessments}

Behavioral assessments began after 3 cisplatin administrations. The animals were tested sequentially in a series of behavioral tests in the following order: (1) open field test (OFT), (2) elevated plus-maze (EPM), (3) Morris water maze (MWM), (4) rat Iowa gambling task (RGT). The rats were given a recovery phase of 5 days before, and 5 days after MWM to maintain a fair general condition during chronic cisplatin treatment. All behavioral procedures were conducted during the light phase of the day. To minimize possible circadian influences on the rats, cisplatin and control rats were observed alternate basis.

Table 2 Experimental design

\begin{tabular}{llll}
\hline $\begin{array}{l}\text { Recent memory } \\
\text { Experimental day }\end{array}$ & Treatments & $\begin{array}{l}\text { Remote memory } \\
\text { Experimental day }\end{array}$ & Treatments \\
\hline $1,2 \ldots 13,14$ & Drug injections & $1,2 \ldots 13,14$ & Drug injections \\
15,16 & Habituation & 15,16 & Habituation \\
17 & OPT, EPM & 17 & OPT, EPM \\
$18,19,20,21,22$ & Recovery phase before MWM & $18,19,20,21,22$ & Recovery phase before MWM \\
$23,24,25,26$ & MWM training & $23,24,25,26$ & Drug injections \\
27 & MWM recent testing & $27,28 \ldots 55,56$ & MWM remote testing \\
$28,29,30,31,32$ & Recovery phase after MWM & 57 & \\
$33,34,35$ & Food restriction for RGT & & \\
$36,37, \ldots 40,41$ & RGT training & & \\
42,43 & RGT testing and Electrophysiological recording & & \\
\hline
\end{tabular}

Cisplatin or saline was administrated on days $1,7,14,21,28,35$ for recent memory group and days $1,7,14,21,28,35,42,49$ for remote memory group 


\section{Open field test (OFT)}

The OFT is often used to assess exploration in a novel environment and offers a preliminary screen for anxietyrelated behavior in rats [19]. Before the actual testing, animals were habituated to the behavioral testing room for 1-2 h sessions on 2 consecutive days. During a 5-min testing, rats were placed individually in the center of a $40 \times$ $40 \mathrm{~cm}$ square drawn in the middle of a black square arena $(80 \times 80 \times 40 \mathrm{~cm})$ and then allowed to explore the field freely. The apparatus was cleaned between rats using $70 \%$ ethanol. Spontaneous exploration behavior in the OFT were observed and recorded by ANY-maze (Stoelting Co., Wood Dale, IL, US), as indicated by total horizontal distance traveled $(\mathrm{m})$, number of rearings (times), time spent (s) and number of entries (times) in the center and peripheral areas, and freezing time (s).

\section{Elevated plus-maze (EPM)}

The EPM is a popular behavioral test for anxiety-like behavior and is thought to result from natural aversion of rats to explore elevated and open areas [20]. The apparatus was made of brown acrylic plastic with two sets of opposing arms (open arms: $50 \times 10 \mathrm{~cm}$; closed arms: $50 \times$ $10 \times 40 \mathrm{~cm}), 50 \mathrm{~cm}$ from the ground. Rats were placed individually in an open field for $5 \mathrm{~min}$ and then allowed to start exploring the maze freely from the junction of the two sets of arms $(10 \times 10 \mathrm{~cm})$ facing one open arm in a 5min test. The maze was cleaned between rats using $70 \%$ ethanol. Time spent in each arm was recorded using the ANY-maze with entries being defined as $85 \%$ of the area of the animal being present in the area entered. Time spent in open arms, especially the percentage of open arm time versus closed arm time was evaluated to assess anxiety.

\section{Morris water maze (MWM)}

The MWM was used to assess and compare spatial learning and memory in cisplatin-treated and control rats as described previously [26, 67]. Experiments were performed in a black circular tank (a diameter of $150 \mathrm{~cm}$ with $60 \mathrm{~cm}$ depth), which was filled with the opaque water $\left(22-24{ }^{\circ} \mathrm{C}\right)$ by adding black nontoxic paint. A circular platform (diameter of $10 \mathrm{~cm}$ and $25 \mathrm{~cm}$ high) submerged $2.0 \mathrm{~cm}$ below water level in the center of one of the four quadrants (target quadrant). Behavioral data of the animal in the water maze were acquired using the ANY-maze by a digital video camera located above the center of the tank.

Four consecutive days of probe trainings were performed to assess special learning. Rats were released from 4 randomized release points facing the tank wall to learn to locate a hidden platform in $60 \mathrm{~s}$ using visual orientation cues on the walls of the tank and on the curtains around the tank. Rats either found the platform by themselves or were guided by the experimenter, and remained on the platform for $10 \mathrm{~s}$. Four trials were conducted each day with intervals of $30 \mathrm{~min}$ between each. Twenty-four hours or thirty days after the last training session, a single probe test was performed to assess recent or remote spatial memory, respectively. The hidden platform was removed to assess memory retention for the submerged platform location and rats were released from the farthest position of original platform and allowed to search the target quadrant that previously contained the escape platform. Swim paths for all trails were recorded and latency to reach platform (sec), distance traveled $(\mathrm{m})$, swimming speed $(\mathrm{m} / \mathrm{s})$, and the amount of time spent in the target quadrant (\% of total time) were calculated to assess spatial recent or remote memory acquisition and retention/retrieval.

\section{Rat lowa gambling task (RGT)}

The rat Iowa gambling task was used to detect decisionmaking. The rats made choices from options associated with different amounts of reward (food pellets) but also different amounts and likelihoods of penalties (timeouts) [18]. The training and test procedures for the rat were identical to the ones previously described by Rivalan et al. [49].

The task was performed in four polyvalent conditioning boxes $(28 \times 30 \times 34 \mathrm{~cm})$ adapted from five-choice serial reaction time chambers (Imetronic, Pessac, France). In each box, there were 4 nose-poke holes illuminated with white LED on the front curved wall, a food dispenser providing food pellets ( $45 \mathrm{mg}$, TestDiet, USA) at the back wall and a transparent central opening partition $(7 \times 7 \mathrm{~cm})$ dividing the box into two chambers at the middle. Infra-red detectors were equipped in holes to detect the nose poke and connected to the food dispenser.

Prior to the actual training, food was restricted over a 3day period following a 1-day fasting period. Simultaneously, 50 reward pellets per rat were put inside the cage every day to make sure the rats became habituated to their taste. During the training phase, daily food was restricted for each rat to about $5 \mathrm{~g}$ of food per $100 \mathrm{~g}$ body weight and rats usually took 5-7 days to make the association between nose-pokes in illuminated holes and food rewards in the food dispenser. In order to guarantee that the selection of the nose poke was a conscious choice, the rats were trained to associate a single nose-poke with one food pellet delivery according to a criterion of at least 100 pellets obtained within a 30-min session, followed by two consecutive nose-pokes with one food pellet delivery with the same criterion. Two final 5-min training sessions were conducted to habituate the rats with the quantity of pellets that could be obtained during the test. The first session was set by two nose-pokes with two pellets at a time (maximum 30 pellets) after making a choice and the other with one pellet (maximum 15 pellets). 
The test procedure was performed the following day and lasted $60 \mathrm{~min}$ or was cut off by 250 pellets obtainment. Rats were free to make choices among the four holes (A-D) as during the training phase; however, different choices were associated with different outcomes set as follows: choices A or B related to two pellets each time as immediate reward, but had separately $1 / 2$ probability to trigger a long penalty time-out (222 s) or $1 / 4$ probability for a very long penalty time-out (444 s), during which period no pellet can be obtained; choices $C$ or $\mathrm{D}$ associated to smaller immediate reward (one pellets each time), but also smaller penalty (1/4 chance for $12 \mathrm{~s}$ time-out, or $1 / 2$ chance for $6 \mathrm{~s}$ time-out). Although the immediate reward of choice $\mathrm{A}$ and $\mathrm{B}$ was twofold that of choice $C$ and $D$, in the long run the choice $C$ or D would allow for the rats to obtain more food pellets compared to choice A or B. Thus the choices $\mathrm{C}$ and D were advantageous choices, while the choices $\mathrm{A}$ and $\mathrm{B}$ were disadvantageous choices. The percentage of advantageous choices (number of nose-pokes $(C+D)$ / number of nose-pokes $(\mathrm{A}+\mathrm{B}+\mathrm{C}+\mathrm{D}) * 100 \%)$ was used as a criterion to distinguish the good ( $>70 \%$ preference of advantageous choices during the last 20 min of RGT test), undecided (30\% - $70 \%$ preference) and poor $(<30 \%$ preference) decision-makers. Among the good decision makers, delayed-good decision-making was determined when the percentage of advantageous choices was below $70 \%$ at $30 \mathrm{~min}$ and above $70 \%$ in the last $20 \mathrm{~min}$.

\section{Electrophysiological recordings}

The electrophysiological recordings in both the control and cisplatin-treated rats were performed after the series of behavioral tests.

\section{Evoked local field potential (LFP) recording}

LFP in the anterior cingulate cortex (ACC) elicited by the electrical stimulation of the basolateral amygdala (BLA) was used as a quantitative measure of synaptic potency in BLAACC pathway. Eleven male Sprague-Dawley rats (5 control and 6 cisplatin-treated rats) were anesthetized (with $1.5 \mathrm{~g} / \mathrm{kg}$ urethane, i.p.) and placed in a stereotaxic frame with body temperature maintained at $36.5 \pm 0.5{ }^{\circ} \mathrm{C}$. A glass recording microelectrode filled with $2.0 \mathrm{M} \mathrm{NaCl}$ was slowly lowered into the ACC (AP 2.0 - $3.8 \mathrm{~mm}$, ML 0.5 - $1.0 \mathrm{~mm}$, depth $1.5-3.5 \mathrm{~mm})$. A bipolar tungsten electrode was placed in the ipsilateral BLA (AP -3.0 to $-3.3 \mathrm{~mm}$, ML $4.8-5.3 \mathrm{~mm}$, depth $6.7-7.5 \mathrm{~mm}$ ). To evaluate synaptic potency test stimuli $(500 \mu \mathrm{A}$, square wave pulse, duration: $0.2 \mathrm{~ms})$ were delivered at $0.033 \mathrm{~Hz}$. After responses stabilized, variations in the stimulus current (from 50-1000 $\mu \mathrm{A}$, increasing $50 \mu \mathrm{A}$ intervals) were delivered in order to generate input-output curves (I/O). The details of the procedure were similar to those of previous publications [33, 68].

\section{Long-term potentiation (LTP) induction}

To examine the synaptic plasticity, LTP was induced by applying theta burst stimulation (TBS, three sets of 10 trains with $10 \mathrm{~s}$ intervals; each train consisted of 10 bursts at $5 \mathrm{~Hz}$ containing 5 pulses at $100 \mathrm{~Hz}$ ) to the BLA. The test stimulate intensity was chosen to evoke $50 \%$ of the maximum amplitude of evoked LFP. Details of the procedure have been described previously [33].

\section{Multiple electrodes recording}

In brief, rats were anesthetized and placed in a stereotaxic frame. Two small holes (1-2 mm wide) were drilled above the BLA and ACC to insert 16-channel silicon-based electrodes (Plexon, Dallas, TX). The silver grounding wires from the electrodes were wrapped around the mounting screws. Electrodes were slowly advanced using a micropositioner until clear neuronal action potentials in most recording channels were observed on-line (OmniPlex D system, Plexon, Dallas, TX). LFPs and spike firings were recorded with a 64channel electrophysiological data acquisition system. LFPs were amplified $(\times 20,000)$, band-pass filtered (0.05 $200 \mathrm{~Hz}, 4$-pole Bessel) and sampled at $1 \mathrm{kHz}$. Spikes were filtered $(0.3-5 \mathrm{kHz}$, 4-pole Bessel) and sampled at $40 \mathrm{kHz}$. Signals from the BLA and ACC were recorded simultaneously during spontaneous activity and visceral stimulation (colorectal distention, CRD, $60 \mathrm{mmHg}$ ) [33].

\section{Histological identification}

After completion of the electrophysiological recordings, a DC current (100-500 $\mu \mathrm{A})$ was passed between the recording electrodes and ground to lesion the brain. The rats were perfused with saline followed by $4 \%$ paraformaldehyde and then the brains were sliced at $50 \mu \mathrm{m}$ and stained with Cresyl violet. Drawings were made of sections showing electrode tracks related to the structure of the ACC and BLA. A standard rat atlas was used as reference for reconstruction of the stimulating and recording sites [69].

\section{Data analysis \\ Statistical analyses}

Data from the behavioral tests were analyzed by ANYmaze, POLY File (Imetronic, Pessac, France), SPSS (IBM SPSS Statistics 20) and GraphPad (Prism 5.0). Data were compared using student's unpaired t-test and one-way and two-way analysis of variance (ANOVA) followed by the Bonferroni post hoc test. Comparisons of proportions of individuals in the gambling task were conducted using the non-parametric Mann-Whitney test. The neural data were processed off-line using NeuroExplorer 5 (Plexon, Dallas, TX) and exported to custom written MATLAB (MathWorks, Natick, MA) programs for additional analysis. Data sets were compared by one-way 
repeated-measures ANOVA followed by multiple comparisons of Bonferroni's test using Prism 5.0 (GraphPad, La Jolla, CA). Results were expressed as mean \pm SE. $\mathrm{P}<$ 0.05 was considered statistically significant.

\section{Spike sorting}

The single-unit spike sorting was performed with OffLine Spike Sorter V3 software (OFSS, Plexon Inc., Dallas, TX) using combined manual and automatic sorting techniques. Spikes were identified when 3 SDs higher than the noise amplitude. All waveforms recorded from each channel were isolated as distinct clusters in 3D space based on the characteristics of spike waveforms using principal component analysis (PCA). Automatic techniques were used to generate separation of waveforms into individual clusters. Manual checking was performed to ensure that the spike waveforms were consistent and cluster boundaries were clearly separated. All isolated single-units exhibited recognizable refractory periods $(\geq 1 \mathrm{~ms})$ in the inter-spike interval (ISI) histograms [70].

\section{Spectral analysis}

Oscillatory brain activity in the theta $(4-10 \mathrm{~Hz})$ frequency range is believed to temporally linked single neuron into assemblies cooperatively facilitate synaptic plasticity [40], and plays a role in many different cognitive functions, including memory and decision making [45]. To clarify alterations in the theta power spectra following cisplatin treatment, we calculated the ACC theta power, peak frequency of the theta oscillations, as well as the theta ratio (theta/(theta + delta). In order to achieve this, the raw ACC LFPs were filtered between 1 and $100 \mathrm{~Hz}$ using noncausal zero-phase-shift filter (fourth-order Butterworth). The power spectral densities (PSD) were characterized using multi-taper estimates with 7 tapers and $2^{13}$ frequency bins (NeuroExplorer 5, Plexon, Dallas, TX). The overlapping percentage of each window is $50 \%$. The power spectra were normalized so that the sum of all the spectrum values equals the mean squared value of the signal. The PSD curve was smoothed with a Gaussian filter (15 bins running average), with the band power being defined as the area under the curve (AUC) of the corresponding frequency domain. The power from each animal was averaged over the 16 channels and to compute the theta/delta ratio, the AUC of the theta band power to the theta plus delta band $(1-10 \mathrm{~Hz})$ was calculated. To expose the time-frequency features of the theta oscillations in both BLA and ACC, the time-varying power spectra were calculated by FFT with Hann window function using NeuroExplorer 5 . The spectrum units were normalized by raw PSD, so that the sum of all the spectrum values equals to the mean squared value of the signal.

\section{The phase-locking of single neuron activity to the theta oscillations of local field potential}

To investigate the angular distributions of spikes in relation to the theta oscillation, and clarify the significance of phase-locking of spikes with the theta oscillation, the phase distribution and Rayleigh test were performed using in house MATLAB scripts modified based on the study in [17]. To ensure the validity of the statistical results, only neurons that had at least 50 spikes were used for phase-locking estimation. Before performing the analysis, 22 different frequencies ranging from 1.6 to $64 \mathrm{~Hz}$ were selected, such that, $\mathrm{f}=2^{\mathrm{x}}$ with $\mathrm{x}=\{6 / 8,8 / 8,10 / 8$, $12 / 8, \ldots, 48 / 8\}$. The LFP was then convolved with a series of Morlet wavelets centered about each of the selected frequencies and each with a length of 4 cycles. The result of these wavelet transforms, is a matrix of vectors whose absolute values (or length) and arguments (or angles) represent the amplitude and phase, respectively, of the LFP at frequency $f$ and time $t$. At each of the selected frequencies, the phase angle of each spike was estimated from the inverse tangent of the real and imaginary components of the wavelet transform. The circular mean of the spike phases was calculated by taking the weighted sum of the cosine and sine of the angles. The mean angle was then the angle of the results and the mean vector length $(R)$ the absolute value over the number of spikes. To check for phase-locking, the Rayleigh test was used to compare against uniformity by calculating the test statistic and a $p$ value. A neuron was considered phase locked in theta range if the $P$ value is below the threshold of 0.0023 which is 0.05 Bonferroni corrected for multiple comparisons $(0.05 / 22$, twenty-two frequencies were tested) [17].

\section{Synchronized theta oscillations between BLA and ACC by cross-correlation analyses}

The synchronized theta activities between the BLA $\operatorname{LFP}_{\theta}$ and ACC $\mathrm{LFP}_{\theta}$ were evaluated by cross-correlograms using NeuroExplorer 5 software. We aligned the $2 \operatorname{LFP}_{\theta} \mathrm{s}$ and chose the BLA LFP ${ }_{\theta}$ as reference. The Pearson correlation values were calculated with a lag time ranging from -0.5 to $0.5 \mathrm{~s}$ with small bins $(2 \mathrm{~ms})$. The cross-correlation curves were smoothed with a Gaussian filter (5 bins running average). We averaged the cross-correlograms from valid electrode channels in the BLA and ACC and took the second positive peak as a quantitative measure because it locates at about $0.2 \mathrm{~s}$ lag time which represents theta activity at about $5 \mathrm{~Hz}[71]$.

\section{Abbreviations}

ACC: Anterior cingulate cortex; BLA: Basolateral amygdala; CRD: Colorectal distension; EPM: Elevated plus-maze; LFP: Local field potential; LTP: Long-term potentiation; MWM: Morris water maze; OFT: Open field test; RGT: Rat lowa gambling task; TBS: Theta burst stimulation. 


\section{Competing interests}

The authors declare that they have no competing interests.

\section{Authors' contributions}

LM: behavioral studies, technical support supervision; analysis and interpretation of data; JW and BC: electrophysiology studies, analysis and interpretation of data; BJ and RC: help analysis of multi-channel data; XX, MH and XZ: behavioral studies helper, interpretation of data and manuscript preparation; $Y L$ : study concept, design, and supervision; analysis and interpretation of data, obtain funding and wrote the manuscript. All authors read and approved the final manuscript.

\section{Acknowledgments}

This work was supported by the Research Grants Council of Hong Kong [reference number: CityU 11100914, 160811, 160812, and 160713 to Y. Li; CityU 123312 to R. Chan], the National Science Foundation of China [81170353 to Y. Li], Health and Medical Research Fund of Hong Kong [reference number: CityU 01122006 to Y. Li] and City University of Hong Kong Neuroscience Research Infrastructure Grant [9610211 to Y. Li]. This work was also supported by the City University of Hong Kong Centre for Biosystems, Neuroscience, and Nanotechnology Grant [9360148 to S. Pang and Y. Li].

We thank Dr. Georges M. Halpern for editing manuscript.

\section{Author details}

'Department of Biomedical Sciences, City University of Hong Kong, Tat Chee Avenue, Kowloon, Hong Kong. ${ }^{2}$ Centre for Biosystems, Neuroscience, and Nanotechnology, City University of Hong Kong, Kowloon, Hong Kong. ${ }^{3}$ Department of Electronic Engineering, City University of Hong Kong, Kowloon, Hong Kong. ${ }^{4}$ Shenzhen Key Lab of Neuropsychiatric Modulation, CAS Center for Excellence in Brain Science, Shenzhen Institutes of Advanced Technology, Chinese Academy of Sciences, Shenzhen 518055, China.

Received: 16 December 2014 Accepted: 14 May 2015

Published online: 23 May 2015

\section{References}

1. Vardy J, Wefel JS, Ahles T, Tannock IF, Schagen SB. Cancer and cancer-therapy related cognitive dysfunction: an international perspective from the Venice cognitive workshop. Annals of oncology: official journal of the European Society for Medical Oncology/ESMO. 2008;19:623-9.

2. Jacobs S, McCully CL, Murphy RF, Bacher J, Balis FM, Fox E. Extracellular fluid concentrations of cisplatin, carboplatin, and oxaliplatin in brain, muscle, and blood measured using microdialysis in nonhuman primates. Cancer Chemother Pharmacol. 2010;65:817-24.

3. Dietrich J, Han R, Yang Y, Mayer-Proschel M, Noble M. CNS progenitor cells and oligodendrocytes are targets of chemotherapeutic agents in vitro and in vivo. J Biol. 2006:5:22.

4. Shabani M, Nazeri M, Parsania S, Razavinasab M, Zangiabadi N, Esmaeilpour K, et al. Walnut consumption protects rats against cisplatin-induced neurotoxicity. Neurotoxicology. 2012;33:1314-21.

5. Husain K, Whitworth C, Hazelrigg S, Rybak L. Carboplatin-induced oxidative injury in rat inferior colliculus. Int J Toxicol. 2003;22:335-42.

6. Rzeski W, Pruskil S, Macke A, Felderhoff-Mueser U, Reiher AK, Hoerster F, et al. Anticancer agents are potent neurotoxins in vitro and in vivo. Ann Neurol. 2004;56:351-60.

7. Ta LE, Espeset L, Podratz J, Windebank AJ. Neurotoxicity of oxaliplatin and cisplatin for dorsal root ganglion neurons correlates with platinum-DNA binding. Neurotoxicology. 2006;27:992-1002.

8. Nokia MS, Anderson ML, Shors TJ. Chemotherapy disrupts learning, neurogenesis and theta activity in the adult brain. Eur J Neurosci. 2012;36:3521-30.

9. Lynch MA. Long-term potentiation and memory. Physiol Rev. 2004;84:87-136.

10. Zhuo M. Long-term potentiation in the anterior cingulate cortex and chronic pain. Philos Trans R Soc Lond B Biol Sci. 2014;369:20130146.

11. Cassell MD, Wright DJ. Topography of projections from the medial prefrontal cortex to the amygdala in the rat. Brain Res Bull. 1986;17:321-33.

12. Gabbott PL, Warner TA, Jays PR, Salway P, Busby SJ. Prefrontal cortex in the rat: projections to subcortical autonomic, motor, and limbic centers. J Comp Neurol. 2005;492:145-77.
13. Porrino LJ, Crane AM, Goldman-Rakic PS. Direct and indirect pathways from the amygdala to the frontal lobe in rhesus monkeys. J Comp Neurol. 1981;198:121-36.

14. Vogt BA. Pain and emotion interactions in subregions of the cingulate gyrus. Nat Rev Neurosci. 2005;6:533-44.

15. Rushworth MF, Behrens TE. Choice, uncertainty and value in prefrontal and cingulate cortex. Nat Neurosci. 2008;11:389-97.

16. Markram H, Lubke J, Frotscher M, Sakmann B. Regulation of synaptic efficacy by coincidence of postsynaptic APs and EPSPs. Science. 1997;275:213-5.

17. Rutishauser U, Ross IB, Mamelak AN, Schuman EM. Human memory strength is predicted by theta-frequency phase-locking of single neurons. Nature. 2010;464:903-7.

18. Rivalan M, Ahmed SH, Dellu-Hagedorn F. Risk-prone individuals prefer the wrong options on a rat version of the lowa Gambling Task. Biol Psychiatry. 2009;66:743-9.

19. Prut $L$, Belzung $C$. The open field as a paradigm to measure the effects of drugs on anxiety-like behaviors: a review. Eur J Pharmacol. 2003:463:3-33.

20. Komada M, Takao K, Miyakawa T. Elevated plus maze for mice. Journal of visualized experiments. 2008;22: e1088. doi: 10.3791/1088.

21. Morris R. Developments of a water-maze procedure for studying spatial learning in the rat. J Neurosci Methods. 1984;11:47-60.

22. Kesner RP, Churchwell JC. An analysis of rat prefrontal cortex in mediating executive function. Neurobiol Learn Mem. 2011;96:417-31.

23. de Visser L, Homberg JR, Mitsogiannis M, Zeeb FD, Rivalan M, Fitoussi A, et al. Rodent versions of the iowa gambling task: opportunities and challenges for the understanding of decision-making. Front Neurosci. 2011;5:109.

24. Bishop SJ. Neurocognitive mechanisms of anxiety: an integrative account. Trends Cogn Sci. 2007;11:307-16.

25. Nakazawa K, McHugh TJ, Wilson MA, Tonegawa S. NMDA receptors, place cells and hippocampal spatial memory. Nat Rev Neurosci. 2004:5:361-72.

26. Teixeira CM, Pomedli SR, Maei HR, Kee N, Frankland PW. Involvement of the anterior cingulate cortex in the expression of remote spatial memory. J Neurosci. 2006;26:7555-64.

27. Frankland PW, Bontempi B, Talton LE, Kaczmarek L, Silva AJ. The involvement of the anterior cingulate cortex in remote contextual fear memory. Science. 2004;304:881-3.

28. Yan N, Cao B, Xu J, Hao C, Zhang X, Li Y. Glutamatergic activation of anterior cingulate cortex mediates the affective component of visceral pain memory in rats. Neurobiol Learn Mem. 2012;97:156-64.

29. Fan J, Wu X, Cao Z, Chen S, Owyang C, Li Y. Up-regulation of anterior cingulate cortex NR2B receptors contributes to visceral pain responses in rats. Gastroenterology. 2009;136:1732-40.

30. Cao Z, Wu X, Chen S, Fan J, Zhang R, Owyang C, et al. Anterior cingulate cortex modulates visceral pain as measured by visceromotor responses in viscerally hypersensitive rats. Gastroenterology. 2008;134:535-43.

31. Gao J, Wu X, Owyang C, Li Y. Enhanced responses of the anterior cingulate cortex neurones to colonic distension in viscerally hypersensitive rats. J Physiol. 2006;570:169-83.

32. Wu X, Gao J, Yan J, Fan J, Owyang C, Li Y. Role for NMDA receptors in visceral nociceptive transmission in the anterior cingulate cortex of viscerally hypersensitive rats. Am J Physiol Gastrointest Liver Physiol. 2008;294:G918-27.

33. Wang J, Zhang X, Cao B, Liu J, Li Y: Facilitation of Synaptic Transmission in the Anterior Cingulate Cortex in Viscerally Hypersensitive Rats. Cereb Cortex 2013. doi: 10.1093/cercor/bht273.

34. Martin SJ, Grimwood PD, Morris RG. Synaptic plasticity and memory: an evaluation of the hypothesis. Annu Rev Neurosci. 2000;23:649-711.

35. Tsvetkov E, Carlezon WA, Benes FM, Kandel ER, Bolshakov VY. Fear conditioning occludes LTP-induced presynaptic enhancement of synaptic transmission in the cortical pathway to the lateral amygdala. Neuron. 2002;34:289-300

36. Sacchetti B, Lorenzini CA, Baldi E, Bucherelli C, Roberto M, Tassoni G, et al. Time-dependent inhibition of hippocampal LTP in vitro following contextual fear conditioning in the rat. Eur J Neurosci. 2002;15:143-50.

37. Varela F, Lachaux JP, Rodriguez E, Martinerie J. The brainweb: phase synchronization and large-scale integration. Nat Rev Neurosci. 2001;2:229-39.

38. Duzel E, Penny WD, Burgess N. Brain oscillations and memory. Curr Opin Neurobiol. 2010;20:143-9.

39. Jacobs J, Hwang G, Curran T, Kahana MJ. EEG oscillations and recognition memory: theta correlates of memory retrieval and decision making. Neuroimage. 2006;32:978-87.

40. Buzsaki G, Draguhn A. Neuronal oscillations in cortical networks. Science. 2004;304:1926-9. 
41. Adamaszek M, Khaw AV, Buck U, Andresen B, Thomasius R. Evidence of neurotoxicity of ecstasy: sustained effects on electroencephalographic activity in polydrug users. PLoS One. 2010;5:e14097.

42. Masuoka T, Kamei $\mathrm{C}$. The role of nicotinic receptors in the amelioration of cholinesterase inhibitors in scopolamine-induced memory deficits. Psychopharmacology (Berl). 2009;206:259-65.

43. Pavlides C, Greenstein YJ, Grudman M, Winson J. Long-term potentiation in the dentate gyrus is induced preferentially on the positive phase of theta-rhythm. Brain Res. 1988;439:383-7.

44. Lisman JE, Idiart MA. Storage of $7+/-2$ short-term memories in oscillatory subcycles. Science. 1995;267:1512-5.

45. Bruno RM, Sakmann B. Cortex is driven by weak but synchronously active thalamocortical synapses. Science. 2006;312:1622-7.

46. Blaisdell AP, Sawa K, Leising KJ, Waldmann MR. Causal reasoning in rats. Science. 2006;311:1020-2

47. Wood JN, Glynn DD, Phillips BC, Hauser MD. The perception of rational, goal-directed action in nonhuman primates. Science. 2007;317:1402-5.

48. Fellows LK, Farah MJ. Different underlying impairments in decision-making following ventromedial and dorsolateral frontal lobe damage in humans. Cereb Cortex. 2005;15:58-63.

49. Rivalan M, Coutureau E, Fitoussi A, Dellu-Hagedorn F. Inter-individual decision-making differences in the effects of cingulate, orbitofrontal, and prelimbic cortex lesions in a rat gambling task. Front Behav Neurosci. 2011:5:22.

50. Rudebeck PH, Walton ME, Smyth AN, Bannerman DM, Rushworth MF. Separate neural pathways process different decision costs. Nat Neurosci. 2006:9:1161-8.

51. Sallet J, Quilodran R, Rothe M, Vezoli J, Joseph JP, Procyk E. Expectations, gains, and losses in the anterior cingulate cortex. Cogn Affect Behav Neurosci. 2007;7:327-36.

52. Kennerley SW, Walton ME, Behrens TE, Buckley MJ, Rushworth MF. Optimal decision making and the anterior cingulate cortex. Nat Neurosci. 2006:9:940-7.

53. Quilodran R, Rothe M, Procyk E. Behavioral shifts and action valuation in the anterior cingulate cortex. Neuron. 2008;57:314-25.

54. Rudebeck PH, Murray EA. Amygdala and orbitofrontal cortex lesions differentially influence choices during object reversal learning. J Neurosci. 2008;28:8338-43

55. de Visser L, Baars AM, Lavrijsen M, van der Weerd CM, van den Bos R. Decision-making performance is related to levels of anxiety and differential recruitment of frontostriatal areas in male rats. Neuroscience. 2011;184:97-106.

56. de Visser L, van der Knaap $L$, van de Loo AJ, van der Weerd CM, Ohl F, van den Bos R. Trait anxiety affects decision-making differently in healthy men and women: towards gender-specific endophenotypes of anxiety. Neuropsychologia. 2010;48:1598-606.

57. Bechara A, Damasio H, Damasio AR, Lee GP. Different contributions of the human amygdala and ventromedial prefrontal cortex to decision-making. J Neurosci. 1999;19:5473-81.

58. Seidenbecher T, Laxmi TR, Stork O, Pape HC. Amygdalar and hippocampal theta rhythm synchronization during fear memory retrieval. Science. 2003:301:846-50.

59. Perez-Jaranay JM, Vives F. Electrophysiological study of the response of medial prefrontal cortex neurons to stimulation of the basolateral nucleus of the amygdala in the rat. Brain Res. 1991;564:97-101.

60. Ji G, Sun H, Fu Y, Li Z, Pais-Vieira M, Galhardo V, et al. Cognitive impairment in pain through amygdala-driven prefrontal cortical deactivation. J Neurosci. 2010;30:5451-64

61. Hoffmann LC, Berry SD. Cerebellar theta oscillations are synchronized during hippocampal theta-contingent trace conditioning. Proc Natl Acad Sci U S A. 2009;106:21371-6.

62. Adhikari A, Topiwala MA, Gordon JA. Synchronized activity between the ventral hippocampus and the medial prefrontal cortex during anxiety. Neuron. 2010;65:257-69.

63. Tesche CD, Karhu J. Theta oscillations index human hippocampal activation during a working memory task. Proc Natl Acad Sci U S A. 2000;97:919-24.

64. Kramis R, Vanderwolf $\mathrm{CH}$, Bland BH. Two types of hippocampal rhythmical slow activity in both the rabbit and the rat: relations to behavior and effects of atropine, diethyl ether, urethane, and pentobarbital. Exp Neurol. 1975:49:58-85.
65. Vandecasteele $M$, Varga $V$, Berenyi A, Papp E, Bartho P, Venance $L$, et al. Optogenetic activation of septal cholinergic neurons suppresses sharp wave ripples and enhances theta oscillations in the hippocampus. Proc Natl Acad Sci U S A. 2014;111:13535-40

66. Ly S, Pishdari B, Lok LL, Hajos M, Kocsis B. Activation of 5-HT6 receptors modulates sleep-wake activity and hippocampal theta oscillation. ACS Chem Neurosci. 2013;4:191-9.

67. Vorhees CV, Williams MT. Morris water maze: procedures for assessing spatial and related forms of learning and memory. Nat Protoc. 2006;1:848-58

68. Maroun M, Richter-Levin G. Exposure to acute stress blocks the induction of long-term potentiation of the amygdala-prefrontal cortex pathway in vivo. J Neurosci. 2003;23:4406-9.

69. Paxinos G, Watson C. The rat brain. 7th ed. San Diego (CA): Academic; 2014.

70. Steenland HW, Li XY, Zhuo M. Predicting aversive events and terminating fear in the mouse anterior cingulate cortex during trace fear conditioning. J Neurosci. 2012;32:1082-95.

71. Jeon D, Kim S, Chetana M, Jo D, Ruley HE, Lin SY, et al. Observational fear learning involves affective pain system and Cav1.2 Ca2+ channels in ACC. Nat Neurosci. 2010;13:482-8.

\section{Submit your next manuscript to BioMed Central and take full advantage of:}

- Convenient online submission

- Thorough peer review

- No space constraints or color figure charges

- Immediate publication on acceptance

- Inclusion in PubMed, CAS, Scopus and Google Scholar

- Research which is freely available for redistribution 